\title{
Neoclassical Transport Coefficients for Tokamaks with Bean-Shaped Flux Surfaces
}

\author{
C.S. Chang*and S.M. Kaye ${ }^{\dagger}$
}

\begin{abstract}
Simple analytic representations of the neoclassical transport coefficients for indented flux surfaces are presented. It is shown that a transport coefficient for an indented flux surface can be expressed in terms of a linear combination of the previously known transport coefficients for two nonindented flux surfaces. Numerical calculations based on actual equilibria from the PBX-M tokamak ${ }^{1}$ indicate that, even for modestly indented flux surfaces, the ion neoclassical thermal transport can be over a factor of two smaller than in a circular plasma with the same midplane radius or with the equivalent area.
\end{abstract}

-Courant Institute of Mathematical Sciences, New York University, 251 Mercer Street, New York, NY 10012, also at Korean Advanced Institute of Science and Technology -

†Princeton Plasma Physics Laboratory, Princeton University, Princeton, N.J. 08543 


\section{Introduction}

A bean-shaped flux surface in a tokamak plasma is characterized by the existence of a toroidally-inside indentation, creating a local minimum in the magnetic field strength $B$ at a poloidal angle where a maximum in $B$ would have been found without indentation. Plasma transport in a bean-shaped flux surface geometry can be peculiar in that two maximum- $B$ locations can exist on a flux surface, and thus two physically separated classes of trapped particles exist, one located outside and the other located inside toroidally, with different detrapping collision rates.

For the system described above, the existing simple analytic expressions for the multi-collisionality neoclassical transport coefficients, such as those given in Refs. 2,3 , and 4, are not readily applicable, since an underlying assumption of these studies is the existence of orly one maximum- $B$ location on any flux surface.

Some studies on extending neoclassical theory beyond that appropriate for standara non-indented cross-sectional plasmas have been carried out. The numerical work of Crume et al. ${ }^{5}$ examined the neoclassical fluxes for two types of shapes, a small aspect ratio, D-shaped plasma, and an indented plasma. In the first case, the reduction in the poloidal variation of $|\mathbf{B}|$ on the outer flux surfaces led to a local reduction in neoclassical fluxes. The authors concluded from ineir numerical calculations that, for the PBX-like plasmas, existing analutic theory of neoclassical transport could still be used even 
with multiple trapped species. An extension of this work was carried out by Beasley and Hogan, ${ }^{6}$ who numerically studied a wider class of plasma shapes as well as the scaling of the neoclassical fluxes with aspect ratio, beta, and collisionality. They indicated that differences between neoclassical transport coefficients between $a$ bean and circle of equivalent midplane radius could be up to a factor of three for $R / a=2.5$.

It is the purpose of this work to develop simple analytic expressions for the neoclassical transport coefficients which can easily be used for unconventional shapes such as an indented plasma.

\section{Analytic Formulation}

The coordinate system $(\psi, \theta, \zeta)$ we choose here is defined as follows: ${ }^{2} \psi$ and $\zeta$ represent the usual poloidal flux and toroidal angle, respectively, and $\theta$ is a poloidal angle defined in such a way that

$$
\vec{B} \cdot \nabla \theta=\vec{B}_{p} \cdot \nabla \theta=k
$$

where $k$ is independent of $\theta$ and $\zeta$. It can be easily seen that $\theta$ can be obtained from the relation

$$
\theta=k \int_{0}^{\ell_{p}} \frac{d \ell_{p}}{B_{p}}
$$

where the integration path is along the poloidal projection of a field line, and $k$ is determined from the requirement that $\theta$ is increased by $2 \pi$ on one 
poloidal circuit:

$$
k=2 \pi / \oint \frac{d \ell_{p}}{B_{p}} .
$$

With this choice of poloidal angle, the flux surface average is simply defined as

$$
<A>=\oint \frac{d \theta}{2 \pi} A .
$$

Notice here that the present choice of poloidal angle may not allow the field lines to be straight in $(\psi, \theta, \zeta)$-space, but this choice is advantageous here because it allows simpler algebraic description of the problem as can be seen in the simplified expression for the flux surface average in Eq. (2).

A neoclassical transport coefficient, $D$, is a flux-surface averaged quantity over local particle or heat flux, $F$, driven by a corresponding driving force of unit magnitude. This velocity-integrated local particle or heat flux $F$ can be a function of local macroscopic quantities like particle density $n$, pressure $p$. plasma flow speed $u$, and the local magnetic field strength $B$. In a tokamak plasrna, the inhomogeneity of a macroscopic physical quantity on a flux surface is caused by the inhomogeneity in $B$ (usually the variation of $n, p$, and $u$ on a flux surface is small). Thus the variation of $F$ on a flux-surface can be simply described as a function of $B$. We introduce a geometry factor $h=B_{0} / B$, where $B_{0}$ is a representative value for $B$ on the flux-surface. Then, $D$ can be writter as

$$
D=\oint \frac{d \theta}{2 \pi} F(h) .
$$

In the banana regime, this $D$ is proportional to the banana factors $I_{i j}$ 's given 
in Ref. 2 , and in the collisional regime it is proportional to the familiar factor $<h^{2}-1 /<h^{-2}>>$. Since $D$ for a nonindented flux surface geometry is well known, we distinguish it by giving a different name $D_{0}$.

Considering $B$ variation in $\theta$, an indented flux-surface is different from an unindented one in that $B$ has two local minima while a non-bean shaped fluxsurface has a single minima of $B$ in $\theta$. A local minimum in $B$ creates a locally trapped group of particles, causing the neoclassical banana enhancement of plasma transport in the weakly collisional case. In an indented geometry, we have two groups of trapped particles with different physical properties (the major and minor radii, average gyroradii, and detrapping rates are different). Their banana-plateau transitions occur at different collision rates, and their contribution to transport is different. Using a rormalized magnetic moment parameter

$$
\lambda^{\prime}=\frac{\mu B_{0}}{\omega},
$$

where $\mu$ is the magnetic moment and $\omega$ is the kinetic energy, the two different trapped regions are described in Fig. 1 in $\lambda^{\prime}-\theta$ space.

Since these two groups of trapped particles are physically separated and have different physical properties, we may divide the poloidal angle domain into two sub-domains in such a way that $B$ has one minimum in $\theta$ in each of the sub-domain $\mathbf{a}$ and $\mathbf{b}$, with the boundaries defined at the values $\theta= \pm \theta_{c}$ corresponding to the poloidal location of maximum $B$ values:

$$
\text { Subdomain a for } \quad-\theta_{c} \leq \theta \leq \theta_{c}
$$




$$
\text { Subdomain } \mathbf{b} \text { for } \theta_{c}<\theta<2 \pi-\theta_{c} \text {. }
$$

Since our flux-surface-average integration is simply a linear combination of the contributions from different spatial locations $\theta$, the quantity $D$ can be written as a linear summation over two subdomains:

$$
\begin{aligned}
D & =D_{a}+D_{b}, \\
D_{a} & =\frac{1}{\pi} \int_{0}^{\theta_{c}} d \theta F_{a}(h), \\
D_{b} & =\frac{1}{\pi} \int_{\theta_{c}}^{\pi} d \theta F_{b}(h),
\end{aligned}
$$

where the up-down symmetry of the flux-surface is assumed.

The difference between $D_{a}$ - or $D_{b}$-integration and $D_{0}$-integration, defined for a nonindented flux-surface, is isi that the integration limits in $D_{n}$ are between 0 and $\pi$ while it is not so for $D_{a}$ and $D_{b}$. However, the limits of the $\theta$-integrations in both $D_{a}$ and $D_{b}$ can be easily extended to 0 and $\pi$ by simple transformation of variables; for $D_{a}$ we need $\theta^{\prime}=\pi \theta / \theta_{c}$, and for $D_{b}$ we need $\theta^{\prime}=\pi\left(\theta-\theta_{c}\right) /\left(\pi-\theta_{c}\right)$. Using this transformation, it is trivial to obtain

$$
\begin{gathered}
D_{a}=\frac{\theta_{c}}{\pi} D_{0}(a), \\
D_{b}=\frac{\pi-\theta_{c}}{\pi} D_{0}(b),
\end{gathered}
$$

where $D_{0}(a)$ and $D_{0}(b)$ are the transformed integrals:

$$
D_{0}(a)=\frac{1}{\pi} \int_{0}^{\pi} d \theta^{\prime} F_{a}(h),
$$




$$
D_{0}(b)=\frac{1}{\pi} \int_{0}^{\pi} d \theta^{\prime} F_{b}(h)
$$

We have divided $D$ into two quantities, $D_{0}(a)$ and $D_{0}(b)$, each $r$ which shows one maximum $h$-value in the transformed poloidal angle $\theta^{\prime}$, which now extends from 0 all the way to $\pi$. Thus, the integrals $D_{0}(a)$ and $D_{0}(b)$ are mathematically indistinguishable from the usual $D=D_{0}$ for a nonindented flux-surface geometry. Previous formulations of the inalytic approximation to $D_{0}$ can be applied to obtain simple analytic representations for $D_{0}(a)$ and $D_{0}(b)$.

The quantity $D$ can then be expressed as

$$
D=D_{a}+D_{b}=\frac{\theta_{c}}{\pi} D_{0}(a)+\frac{\pi-\theta_{c}}{\pi} D_{0}(b)
$$

This simple relation provides a general approximate formula for all the neoclassical transport coefficients in a bean shaped tokamak. It means that we can regard a bean shaped cross-section as a weighted linear combination of two nonindented cross-sections properly transformed by the relation between $\theta$ and $\theta^{\prime}$, with the major radius, minor radius, and $B_{0}$ separately defined in each of the two.subdomains (see Fig. 2), and with the weights allocated according to the $\theta$-widths occupied by each subdomain.

In applying Eq. (3) to the bean shaped flux surfaces, it is convenient to define the horizontal inverse-aspect ratios $\epsilon_{a}$ and $\epsilon_{b}$ for each subdomain as follows:

$$
\epsilon_{a}=\frac{R_{a, \max }-R_{\min }}{2 R_{a 0}}, \quad \epsilon_{b}=\frac{R_{b, \max }-R_{\min }}{2 R_{b 0}}
$$


Here, $R_{a, \max }$ and $R_{b, \max }$ are the distances from the major axis to the minimum$B$ points in the domain $\mathbf{a}$ and $\mathbf{b}$, respectively; $R_{\min }$ is the distance from the major axis to the point $\theta=\theta_{c}$ on the flux surface (see Fig. ' $\dot{z}$ ); and $R_{s 0}=\left(R_{s, \max }+R_{\min }\right) / 2, s=a$ or $b$, defines the horizontal center of each subdomain. Each subdomain has different representative values $B_{a 0}$ and $B_{b 0}$ for the magnetic field strength defined at the horizontal centers.

An example is appropriate at this point to demonstrate the actual application of Eq. (3). The ion thermal conductivity in an unindented geometry can be described by ${ }^{3}$

$$
\left(\beta_{2}, g_{2 i}\right)_{0}=<D_{0}>=-\frac{l^{2}}{|\nabla \psi|^{2}} \frac{n_{i} \rho_{i}^{2}}{\tau_{i}} \epsilon^{1 / 2} K_{2}
$$

where $n_{i}$ is the ion density, $\tau_{i}$ is the ion-ion $90^{\circ}$-collision time, $\rho_{i}$ is the gyroradius at $B=B_{0},(I /|\nabla \psi|) \rho_{i}$ defines the poloidal gyroradius $\rho_{\theta i}$, and $K_{2}$ is

$$
K_{2}\left(\epsilon, \nu_{*}(\epsilon)\right)=K_{2}^{(0)}\left(\frac{\dot{K}_{2} / 0.66}{1+a_{2} \nu_{*}^{1 / 2}+b_{2} \nu_{*}}\left\langle\frac{B_{0}^{2}}{B^{2}}\right\rangle+\frac{\left(c_{2}^{2} / b_{2}\right) \nu_{*} \epsilon^{3 / 2}}{1+c_{2} \nu_{*} \epsilon^{3 / 2}} H_{P} F\right) .
$$

Here, $\nu_{*}$ is the normalized ion banana collision frequency parameter $\mu_{* i}$ of Ref. 3 with the inclusion of impurity effect, $K_{2}^{(0)}=0.66, a_{2}=1.03, b_{2}=$ $0.31, c_{2}=0.74$, and

$$
\begin{gathered}
\hat{K}_{2}=\left[0.66(1+1.54 \alpha)+\left(1.88 \epsilon^{1 / 2}-1.54 \epsilon\right)(1+3.75 \alpha)\right] \\
H_{P}=1+1.33 \frac{\alpha(1+0.60 \alpha)}{1+1.790} \\
F=\frac{1}{2} \epsilon^{-1 / 2}\left(\left\langle\frac{B_{0}^{2}}{B^{2}}\right\rangle-\left\langle\frac{B^{2}}{B_{0}^{2}}\right\rangle^{-1}\right)
\end{gathered}
$$




$$
\begin{aligned}
\left\langle\frac{B^{2}}{B_{0}^{2}}\right\rangle^{-1} & =\frac{\left(1-\epsilon^{2}\right)^{1 / 2}\left(1+\epsilon R_{0}^{\prime} / 2\right)}{1+\left(R_{0}^{\prime} / \epsilon\right)\left[\left(1-\epsilon^{2}\right)^{1 / 2}-1\right]} \\
\left\langle\frac{B_{0}^{2}}{B^{2}}\right\rangle & =\frac{1+\frac{3}{2}\left(\epsilon^{2}+\epsilon R_{0}^{\prime}\right)+\frac{3}{8} \epsilon^{3} R_{0}^{\prime}}{1+\frac{1}{2} \epsilon R_{0}^{\prime}}
\end{aligned}
$$

with $\alpha=Z_{I}^{2} n_{I} / Z_{i}^{2} n_{i}\left(Z_{I}\right.$ and $Z_{i}$ are the impurity and main ion charge numbers, ind $n_{I}$ and $n_{i}$ are the impurity and main ion density). The $\nu_{*}$ dependence is shown explicitly on the left-hand side of Eq. (4) to emphasize the fact that $\nu_{*}$ is a function of $\epsilon$. Applying Eq. (3) to this ion thermal conductivity formula, we have

$$
\left(\beta_{2}, g_{2 i}\right)=-\frac{I^{2}}{|\nabla \psi|^{2}} \frac{n_{i}}{\tau_{i}}\left[\left(\frac{\theta_{c}}{\pi}\right) \epsilon_{a}^{1 / 2} \rho_{i a}^{2} K_{2}\left(\epsilon_{a}, \nu_{* a}\right)+\left(\frac{\pi-\theta_{c}}{\pi}\right) \epsilon_{b}^{1 / 2} \rho_{i b}^{2} K_{2}\left(\epsilon_{b}, \nu_{* b}\right)\right],
$$

where the variables $\left\langle B_{0}^{2} / B^{2}\right\rangle, H_{p}$, and $F$ are evaluated with proper identification of a proper representative $B_{0}$ value $\left(B_{0}=B_{a 0} 0^{-} B_{b 0}\right)$, the horizontal inverse aspect ratio $\epsilon=\epsilon_{a}$ or $\epsilon_{b}, R_{0}=R_{a 0}$ or $R_{b 0}$, and $R_{0}^{\prime}=d R_{0} / d r=R_{a i j}^{\prime}$ or $R_{b 0}^{\prime}$ in each $\theta$-subdomain. Notice here that $\rho_{i}$ is transformed into $\rho_{i a}$ or $\rho_{i b}$ due to the change in the reference magnetic field strength,

$$
\rho_{i}^{2}\left\langle\frac{B_{0}^{2}}{B^{2}}\right\rangle=\rho_{i}^{2} \frac{B_{0}^{2}}{B_{a 0}^{2}}\left\langle\frac{B_{a 0}^{2}}{B^{2}}\right\rangle=\rho_{i \alpha}^{2}\left\langle\frac{B_{a 0}^{2}}{B^{2}}\right\rangle .
$$

The application of Eq. (3) to the ion thermal conductivity has been quite simple because of the fact that all the quantities $K_{2},<B_{0}^{2} / B^{2}>, H_{p}$, and $F$ are well represented by the horizontal aspect ratio and $R_{0}^{\prime}$ only, which are independent of the detailed shape information of the flux surfaces (such as elongation and triangularity). Without this nice feature, the conversion formula could have been more complicated than the one given by Eq. (5), with 
all the extra shaping information in the arguments. The shaping information only came in through the common factor $I /|\nabla \psi|$. This feature is generally true for all the neoclassical transport coefficients, and thus the application of Eq. (3) to all other transport coefficients is expected to be equally simple and easy.

\section{Numerical Comparison}

In order to determine the effect of the two trapping regions on the ion neoclassical thermal diffusivity, we have used actual experimental equilibria and profiles from a bean-shaped plasma produced in the PBX-M tokamak as a basis for a comparison between indented and non-indented shapes. The equilibrium used was of a $\left\langle\beta_{t}\right\rangle \simeq 6.3 \%$ discharge with $I_{p}=0.55 M A, B_{t}=$

$1.1 \mathrm{~T}, P_{\text {inj }}=4.5 \mathrm{MW}, R_{0} \simeq 1.64 \mathrm{~m}$, and $a_{\text {mid }} \simeq 0.28 \mathrm{~m}$. Figure 3 shows the flux surface indentation and $\theta_{c}$ as a function of midplane radius $r_{m}$. Here, the indentation is defined as $\frac{R_{b \text { max }}-R_{\min }}{R_{a_{1} i \text { iax }}-R_{\operatorname{mo1}}}$. The flux surfaces exhibit no indentation up to $r_{\text {mid }}=10 \mathrm{~cm}$; beyond this radius, the indentation increases with $r_{\text {mid }}$, rearhing 0.24 at the plasma surface. This value is slightly less than the maximum indentation achieved in PBX-M (0.28). The value of $\theta_{c}$ is determined from the location off the midplane at which the major radial component of the magnetic field, $B_{R}$, changes sign.

Up to now, it has been very difficult to know just how to estimate analytically the ion neoclassical thermal diffusivity (or neoclassical resistivity) 
for bean-shaped plasmas. Several approaches can be taken, but most involve assuming a circular shape for the plasma. For instance, one can approximate the bean by a "small" circle whose diameter is equal to the bean waist width, a "big" circle whose diameter is given by $R_{a, \max }-R_{\min }$ (see Fig. 2), or an equivalent area circle whose diameter is given by $2 \sqrt{\frac{\pi}{A}}$, where $A$ is the cross-sectional area of each flux surface for the bean-shaped plasma. Figure 4 exhibits the qualitative relationships of these shapes to one another. For comparison purposes, we will calculate the neoclassical factors for the above three shapes in addition to the bean.

For the calculation, appropriate choices of major and minor radii, and normalization factors have to be made. The normalization factor, $f_{s}$, quantifies the poloidal extent of each subdomain. For instance, for region a, $f_{s}=\frac{\theta_{s}}{\pi}$, for region $\mathrm{b}, f_{\mathrm{a}}=\frac{\pi-\theta_{c}}{\pi}$, and for circles, $f_{\mathrm{a}}=1$. Table I gives the information for each of the shapes considered in the calculation. In the table, $R_{0}$ is the geometric center of each flux surface, $a$ is the bean waist width, and $r_{a}, r_{b}, R_{a_{0}}$, $R_{b_{0}}$, and $a_{e q}$ have been defined earlier. The big circle approximation was used for the non-indented flux surfaces for the bean case $\left(r_{\text {mid }} \leq 10 \mathrm{~cm}\right)$.

Figure 5 shows the results of the calculation described above. Plotted on the $\hat{y}$-axis are the values of the ion neoclassical factor, $f_{s} \rho_{i,}^{2} \epsilon_{a}^{1 / 2} K_{2 \downarrow}$, calculated using the minor and major radii, ion gyroradius, effective collision frequency, and normalization factors appropriate for each shape. For the bean, the sum - of the ion neoclassical factors in regions $\mathbf{a}$ and $\mathbf{b}$ is plotted. Several features can be noted in Fig. 5. First of all, for the bean case, the trapping region 
dominating the value of the neoclassical factor is region $a ; \frac{\left(\pi-\theta_{c}\right) \rho_{i_{b}}^{2} e_{b}^{1 / 2} K_{z_{b}}}{\theta_{c} \rho_{i_{a}}^{2} e_{a}^{1 / 2} K_{2_{c}}}$, the ratio of region $b$ to a neoclassical factors, is only several percent maximam. Consequently, since region a dominates the bean case, the difference between the big circle and bean is due only to the difference in normalization factors, 1 versus $\theta_{c} / \pi$, and this difference is only 15 to $20 \%$. The differences among the neoclassical factors of the bean and big circle, and the small and equivalent area circles is due primarily to the difference in choices for minor and major radii of each flux surface (i.e., $\epsilon$ ). As can be seen in the figure, this can lead to over a factor of two difference in the neoclassical factors even at modest indentations.

\section{Summary and Discussion}

In this brief report we have shown how the neoclassical transport coefficients in a bean-shaped geometry can be calculated from standard analytic expressions derived for non-indented flux surfaces. This is accomplished by separating the indented flux surface into two, non-indented regions. The values computed in this fashion can be over a factor of two less than those calculated for a circle with similar minor and major radius. The reason for this is the effective reduction in dominant trapping region for the bean geometry.

This reduction in neoclassical transport may also apply to extremely D-shaped plasmas such as those produced in DIII-D and JET, and those 
planned tor CIT and Alcator C-MOD. The more D-shaped the plasma is, the less trapping will occur on the inside. In a true D-shaped plasma, where the entire inside of the plasma is at the maximum- $B$ locai ion, $\theta_{c}$ can be taken to be the first point off the midplane at which $\partial B / \partial \theta=0$. Consequently, the neoclassical transport coefficient for a D-shaped plasma will be determined using "region a" parameters, which, a" shown in Fig. 5, can result in a significant reduction from the value calculated for a circle. A properly treated transport calculation in a D-shaped plasına should contain this feature.

\section{Acknowledgements}

This work was supported by the U.S. Department of Energy under Grant No. DE-FG02-86-ER5-3223, and Contract No. DE-AC02-76-CHO-3073. 


\section{References}

${ }^{1}$ R.E. Bell, N. Asakura, S. Bernabei, M.S. Chance, P.-A. Duperrex, R.J. Fonck, G.M. Gammel, G. Greene, R.E. Hatcher, A. Holland, S.C. Jardin, T.-W. Jiang, R. Kaita, S.M. Kaye, C.E. Kessel, H.W. Kugel, B. LeBlanc, F.M. Levinton, M. Okabayashi and M. Ono, S.F. Paul, E.T. Powzll, Y. Qin, D.W. Roberts, N.R. Sauthoff, S. Sesnic and H. Takahashi, Phys. Fluids B 2,1271 (1990).

${ }^{2}$ F.L. Hinton and R.D. Hazeltine, Rev. Mod. Phys. 48, 239 (1976).

${ }^{3}$ C.S. Chang and F.L. Hinton, Phys. Fluids 29, 3314 (1986).

${ }^{4}$ S.P. Hirshman and D.J. Sigmar, Nucl. Fusion 21, 1079 (1981).

${ }^{5}$ E.C. Crume Jr., C.O. Beasley Jr., S.P. Hirshman and W.I. van Rij, Phys. Fluids 30, 1152 (1987).

${ }^{6}$ C.O. Beasley, J.T. Hogan, Nucl. Fusion 28, 1923 (i 388 ) 


$\begin{array}{lccc}\text { SHAPE } & \text { MINOR RADIUS } & \text { MAIOR RADIUS } & \text { NORMALIZATION } \\ \text { small circle } & a & R_{0} & 1 \\ \text { big circle } & r_{a} & R_{a_{0}} & 1 \\ \text { eq. area circle } & a_{e q} & R_{0} & 1 \\ \text { bean } & r_{a}, r_{b} & R_{a_{0}}, R_{b_{0}} & \theta_{c} / \pi,\left(\pi-\theta_{c}\right) / \pi\end{array}$

Table I: Geometrical and normalization factors used for shape comparison 


\section{Figures}

FIG. i. Phase space diagram showing the two similarly shaped trapped regions labeled as sut tomains $\mathbf{a}$ and $\mathbf{b}$.

FIG. 2. Poloidal projection of a bean shaped flux surface showing the different major and horizontal minor radii of the two subdomains.

FIG. 3. Indentation (triangles, left ordinate) and $\theta_{c}$ (circles, right ordinate) as a function of $r_{\text {mid }}$ for a high- $\left\langle\beta_{t}\right\rangle$ PBX-M plasma.

FIG. 4. Schematic of various shape definitions used for the comparison.

FIG. 5. Neoclassical factor, $f_{s} \rho_{i}^{2},{ }_{s}^{1 / 2} K_{2,}$, as a function of midplane radius for the four different cases. 
$\# 90 \times 0216$

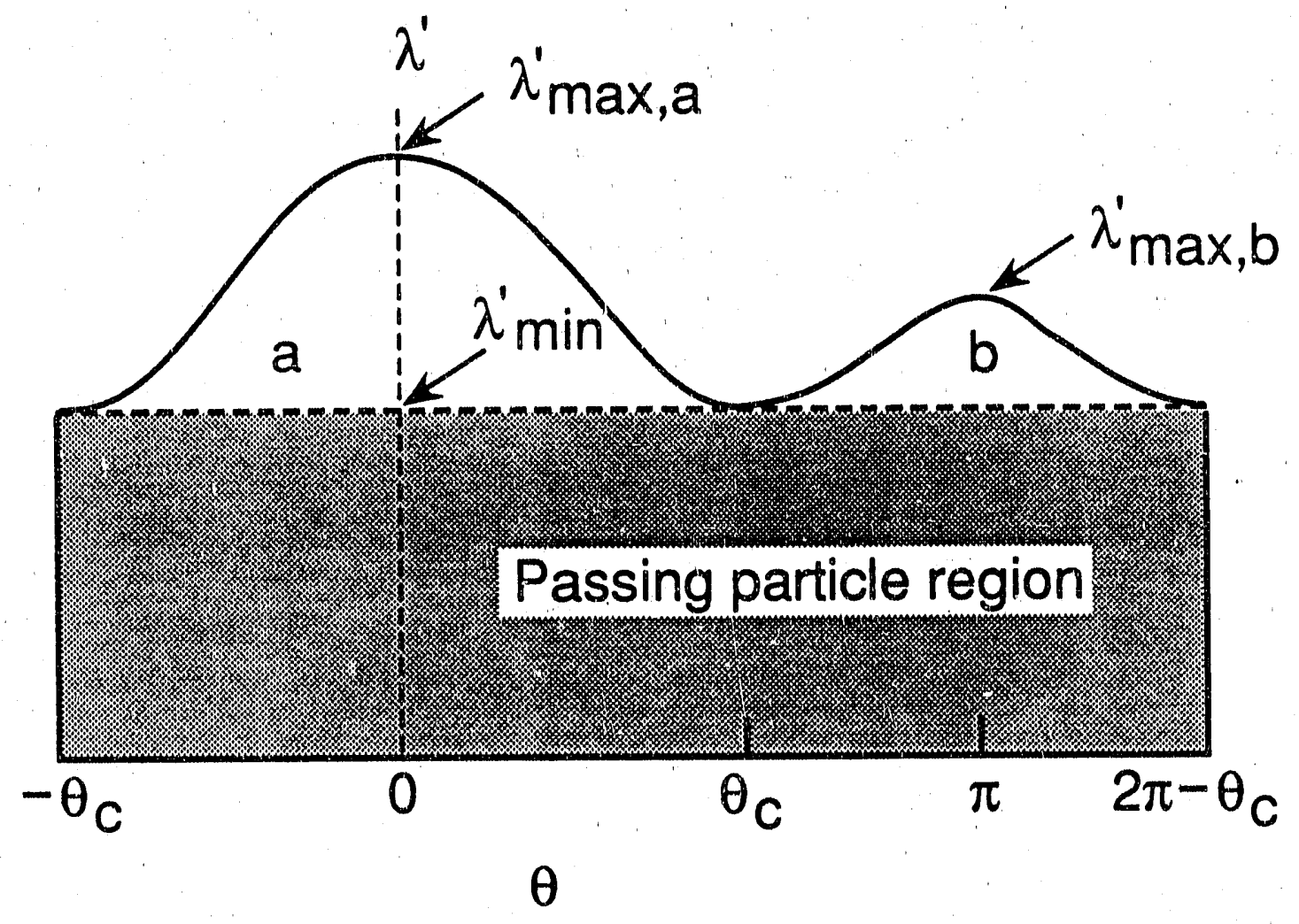

Fig. 1 


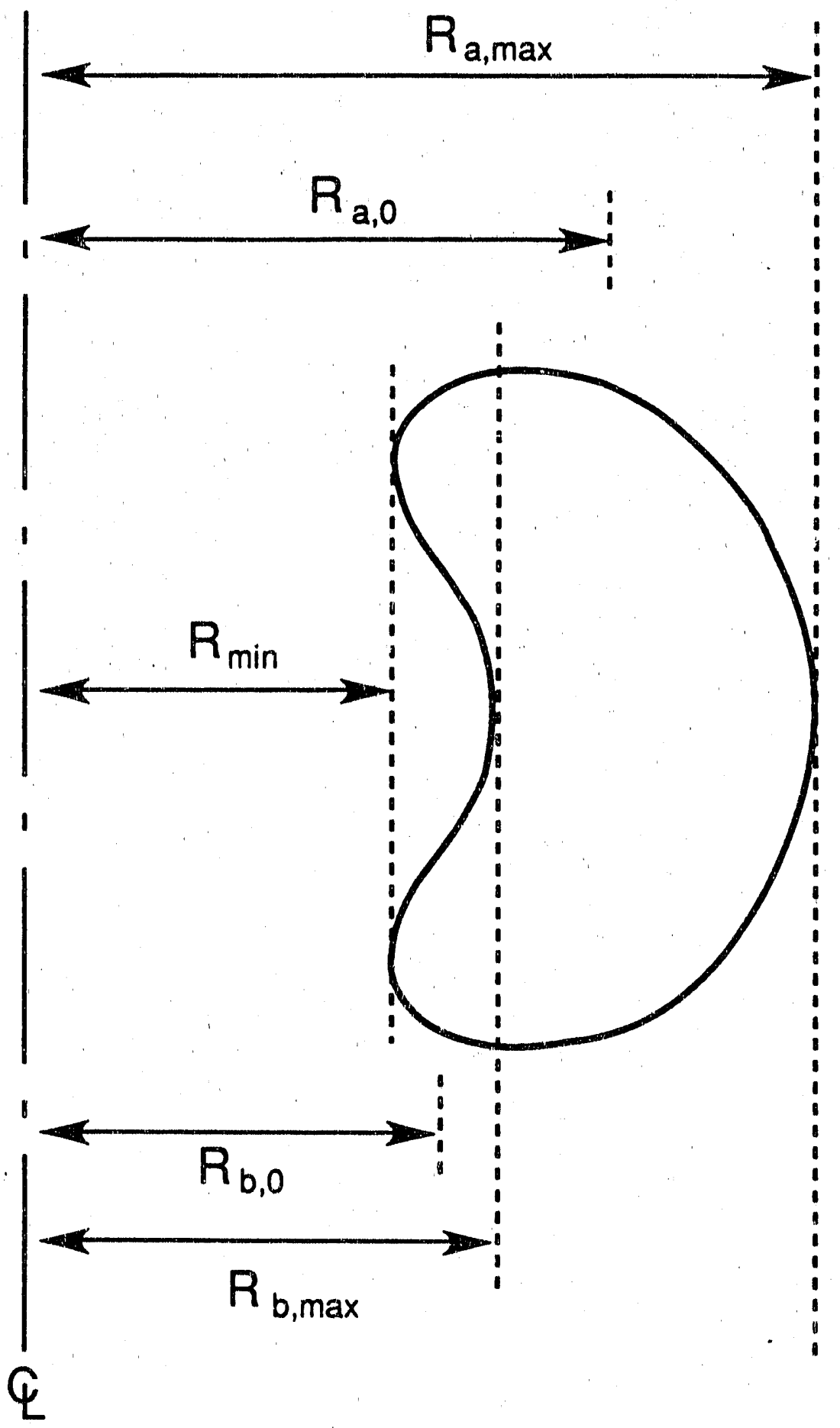

Fig. 2 


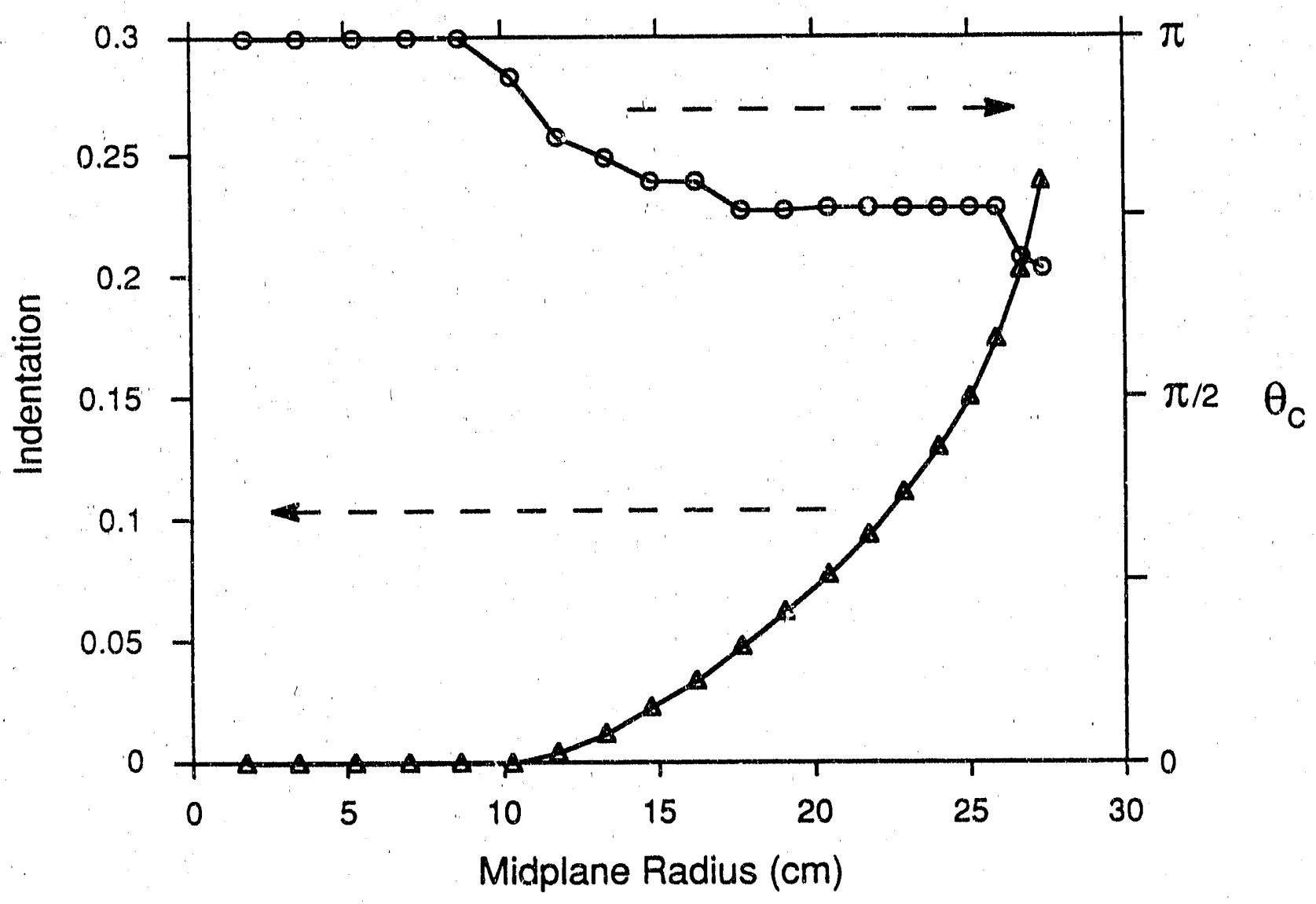

Fig. 3 
\#90X0199

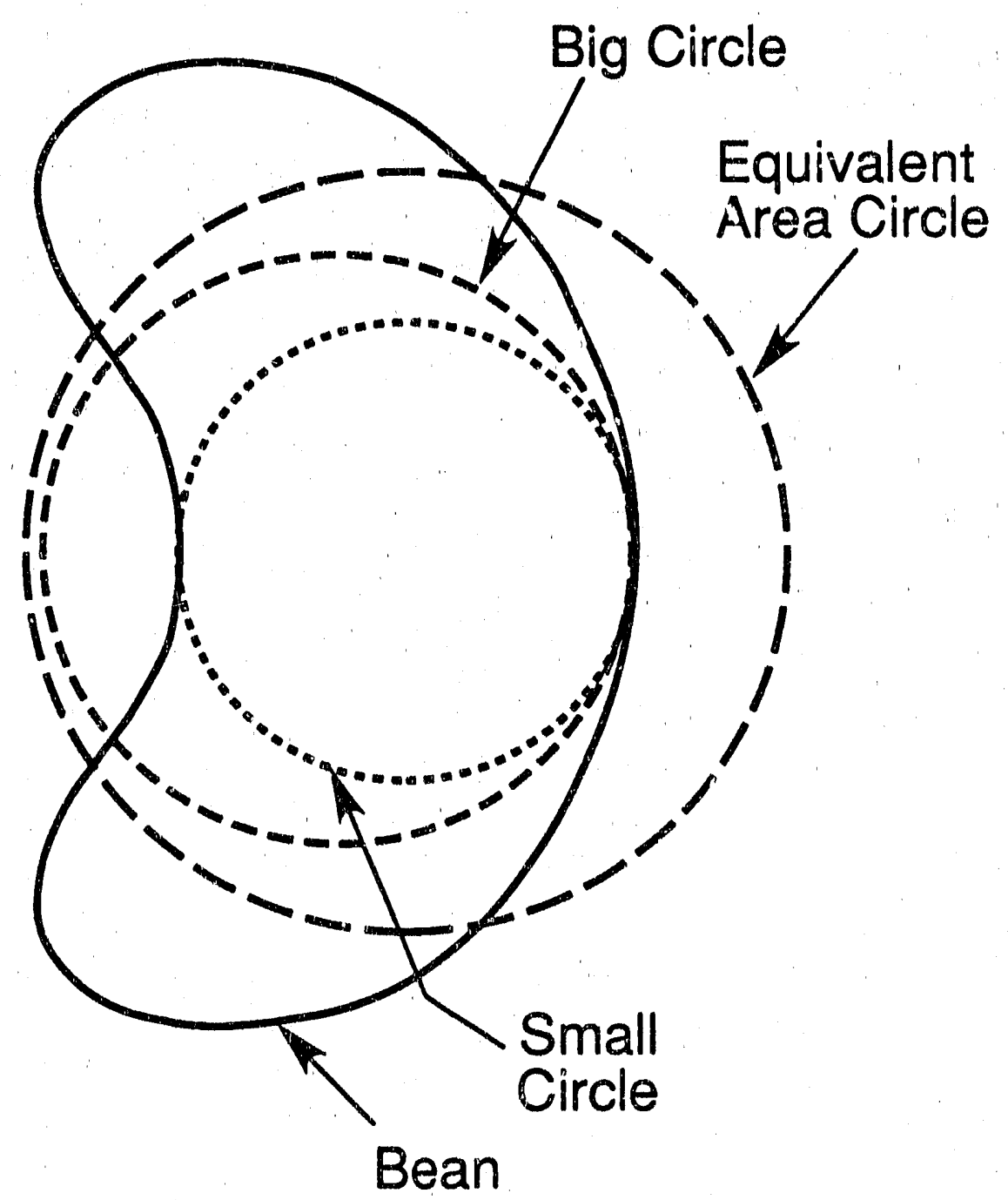

Fig. 4 
\#90X0198

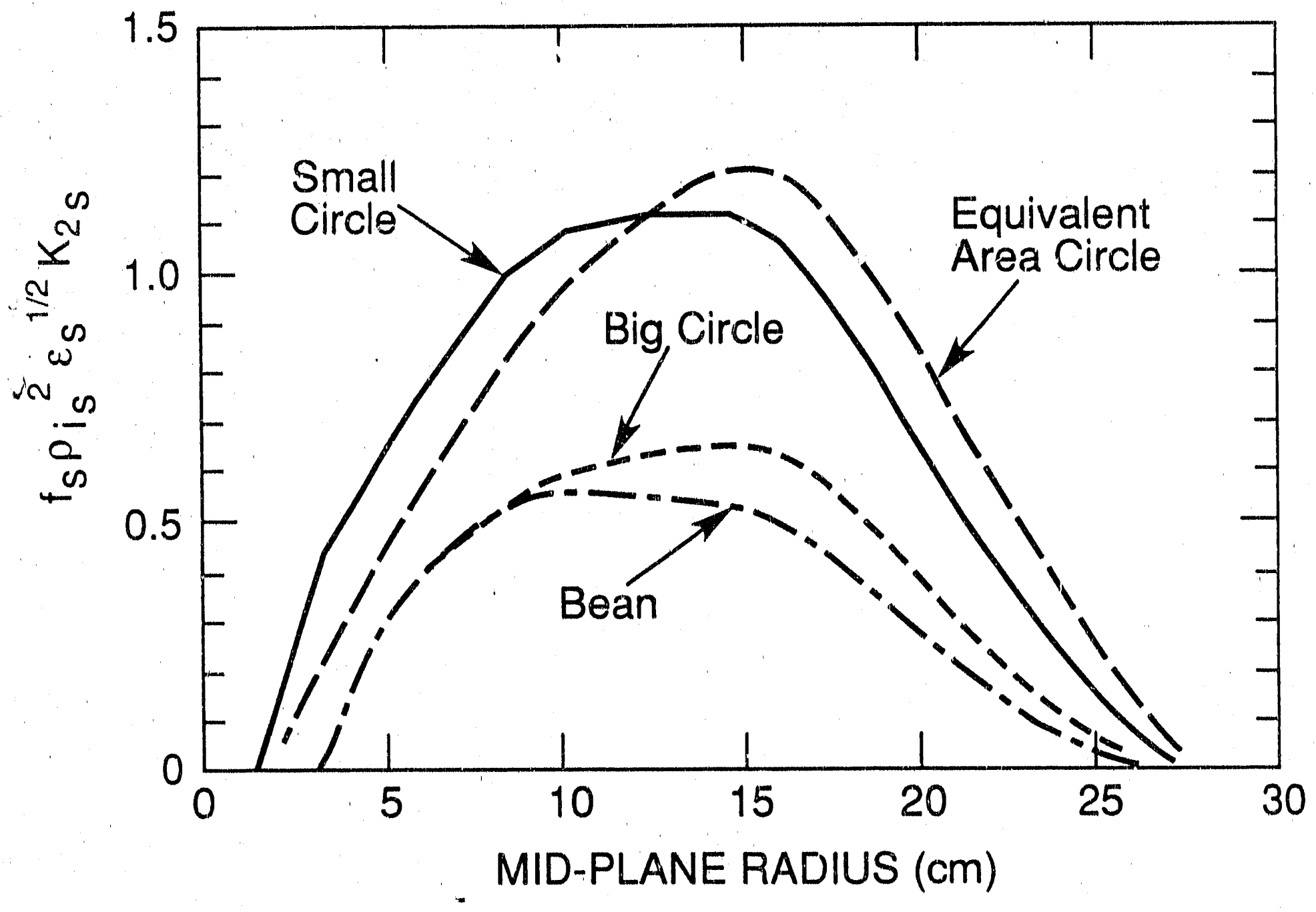

Fig. 5 

